\title{
Fitness advantage and cytoplasmic incompatibility in Wolbachia single- and superinfected Aedes albopictus
}

\author{
SL Dobson, W Rattanadechakul and EJ Marsland \\ Department of Entomology, University of Kentucky, Lexington, KY 40546, USA
}

\begin{abstract}
Wolbachia are obligate, maternally inherited, intracellular bacteria that infect numerous insects and other invertebrates. Wolbachia infections have evolved multiple mechanisms to manipulate host reproduction and facilitate invasion of naive host populations. One such mechanism is cytoplasmic incompatibility $(\mathrm{Cl})$ that occurs in many insect species, including Aedes albopictus (Asian tiger mosquito). The multiple Wolbachia infections that occur naturally in $A$. albopictus make this mosquito a useful system in which to study $\mathrm{Cl}$. Here, experiments employ mosquito strains that have been introgressed to provide genetically similar strains that harbor differing Wolbachia infection types. Cytoplasmic incompatibility levels, host longevity, egg hatch rates, and
\end{abstract}

fecundity are examined. Crossing results demonstrate a pattern of additive unidirectional cytoplasmic incompatibility. Furthermore, relative to uninfected females, infected females are at a reproductive advantage due to both cytoplasmic incompatibility and a fitness increase associated with Wolbachia infection. In contrast, no fitness difference was observed in comparisons of single- and superinfected females. We discuss the observed results in regard to the evolution of the WolbachialA. albopictus symbiosis and the observed pattern of Wolbachia infection in natural populations.

Heredity (2004) 93, 135-142, advance online publication, 5 May 2004; doi:10.1038/sj.hdy.6800458

Keywords: cytoplasmic incompatibility; cytoplasmic drive; population replacement; reproductive parasite; mutualism

\section{Introduction}

Wolbachia are obligate intracellular rickettsia-like bacteria that occur in numerous invertebrates, with infection rate estimates in excess of $20 \%$ of insect species (Werren et al, 1995a; Jeyaprakash and Hoy, 2000; Werren and Windsor, 2000; Jiggins et al, 2001). The success of Wolbachia can be attributed in large part to its ability to manipulate the reproduction of its host to promote infection spread into the host population. Wolbachia infections provide multiple examples of mechanisms that promote infection spread, ranging along the continuum from mutualism (Hoerauf et al, 1999; Langworthy et al, 2000) to reproductive parasitism. Examples of the latter include cytoplasmic incompatibility (Hoffmann and Turelli, 1997), male killing (Hurst et al, 1999), parthenogenesis (Stouthamer et al, 1993), and feminization (Rousset et al, 1992). Although variable in strategy, the multiple mechanisms by which Wolbachia manipulates host reproduction are similar in that they provide infected female hosts with a reproductive advantage relative to uninfected females.

Cytoplasmic incompatibility (CI) results in karyogamy failure and arrested development of early embryos in diploid insects (Tram and Sullivan, 2002). Unidirectional CI occurs in matings between Wolbachia-infected males

Correspondence: SL Dobson. Current address: Department of Entomology; University of Kentucky, Lexington, KY 40546, USA.

E-mail:sdobson@uky.edu

Received 23 September 2003; accepted 27 February 2004; published online 5 May 2004 and uninfected females (Figure 1). The reciprocal cross and matings between individuals that harbor similar Wolbachia infections are compatible. Thus, in host populations that include both infected and uninfected individuals, CI provides a reproductive advantage to infected females since they can mate successfully with all male types. In contrast, uninfected females are incompatible with infected males, reducing reproductive success. The advantage afforded to females by CI comes at the expense of infected males, which are incompatible with uninfected females. However, this is of no cost to Wolbachia, since males are an evolutionary dead end for the maternally inherited infections. As demonstrated both theoretically and empirically, the reproductive advantage afforded by CI to infected females can result in population replacement, with the infected cytotype driving into the host population and replacing the uninfected cytotype (reviewed in Hoffmann and Turelli, 1997).

Wolbachia infections in Aedes albopictus (Asian tiger mosquito) provide a useful system in which to study CI and population replacement. Naturally occurring populations of $A$. albopictus can be single-infected with the wAlbA Wolbachia type or superinfected with the wAlbA and wAlbB Wolbachia types (Kambhampati et al, 1993; Sinkins et al, 1995b; O'Neill et al, 1997; Otsuka and Takaoka, 1997; Dobson et al, 2001). The wAlbA and wAlbB types are within the A and B Wolbachia clades, respectively (Zhou et al, 1998). Prior experiments demonstrate that the $w \mathrm{AlbA}$ infection is unidirectionally incompatible with uninfected hosts, and that the wAl$\mathrm{bA} / w \mathrm{AlbB}$ superinfection has an additive effect, such 


\begin{tabular}{rrrrr} 
& \multicolumn{5}{c}{ Male } \\
Female & $\mathrm{AB}$ & $\mathrm{A}$ & $\mathrm{B}$ & $\mathrm{O}$ \\
$\mathrm{AB}$ & + & + & + & + \\
$\mathrm{A}$ & - & + & - & + \\
$\mathrm{B}$ & - & - & + & + \\
$\mathrm{O}$ & - & - & - & +
\end{tabular}

Figure 1 Generalized crossing pattern illustrating unidirectional and bidirectional cytoplasmic incompatibility ( - ) and compatibility $(+)$ that results in crosses of uninfected $(\mathrm{O})$, single-infected $(\mathrm{A}$ or $\mathrm{B})$, and superinfected (AB) individuals. Note that incompatibility is observed when the male host harbors an infection type that is not present in the host female mate.

that the superinfection is unidirectionally incompatible with both single and uninfected hosts (see Figure 1; Dobson et al, 2001). This pattern of additive unidirectional CI in combination with the observed geographic distribution of single and superinfections in naturally occurring A. albopictus populations (Sinkins et al, 1995b; Perrot-Minnot et al, 1996) has led to the hypothesis that superinfection in $A$. albopictus originated via sequential population replacement events: that the superinfection $(w \mathrm{AlbA}+w \mathrm{AlbB})$ replaced a population that had been previously invaded by the $w$ AlbA single-infection.

Here, we have examined CI levels, longevity, egg hatch, and fecundity in strains of $A$. albopictus (Asian tiger mosquito) that are uninfected, single-infected, and superinfected. Determination of Wolbachia effects can be complicated if the compared strains differ in both Wolbachia infection type and host genetic backgrounds (Dobson et al, 2001). Thus, we compare the differing infection types within a homogeneous host genetic background that was generated via introgression. The results demonstrate that, relative to uninfected females, infected females are at a reproductive advantage due to both cytoplasmic incompatibility and a fitness increase associated with Wolbachia infection. No fitness difference was observed in comparisons of single and superinfected females. A pattern of additive unidirectional cytoplasmic incompatibility was observed, which is consistent with prior reports (Kambhampati et al, 1993; Sinkins et al, 1995b; Otsuka and Takaoka, 1997; Dobson et al, 2001, 2002). We discuss the results in regard to the proposed evolution of Wolbachia superinfection in A. albopictus by sequential population replacement. As host genotype can affect Wolbachia infection dynamics, we compare the experimental results with introgressed strains with additional $A$. albopictus strains that harbor similar infection types but that differ in host genotype.

\section{Materials and methods}

\section{Mosquito stocks and culture}

Mosquito strains and infection types are outlined in Table 1. Mosquitoes were maintained using standard conditions (Gerberg et al, 1994) at $28 \pm 2{ }^{\circ} \mathrm{C}$ and $75 \pm 10 \%$ $\mathrm{RH}$ with an $18 \mathrm{~h}$ light cycle. Eggs were hatched in deoxygenated water and reared at low density in water augmented with liver powder. For adult maintenance

and experimental crosses, a constant supply of $10 \%$ sucrose was provided to adults. Females were provided weekly with a mouse for blood feeding and eggs were collected weekly.

\section{Introgressions and experimental crosses}

Characterization of Wolbachia effects can be complicated by differing host genetic backgrounds (Dobson et al, 2001). In a previous study, genetically homogeneous A. albopictus strains that differed in their infection type were generated via tetracycline treatment (Dobson and Rattanadechakul, 2001; Dobson et al, 2002). However, tetracycline treatment failed to generate a single-infected strain. Here, we have used introgressions to generate genetically homogeneous strains that are uninfected, single-infected (wAlbA) or superinfected $(w \mathrm{AlbA}+w \mathrm{AlbB})$. Introgression crosses were conducted using inbred Hou, Koh, and UjuT mosquito strains (Table 1). The UjuT nuclear genome was introgressed into single- and superinfected cytoplasm types by repeated backcrossing of UjuT males with virgin Koh or Hou females, respectively. In the next generation, the resulting hybrid daughters were mated with UjuT males. The introgression crosses were repeated for a total of seven generations. In theory, each backcross generation will replace half of the maternal nuclear genome with the paternal nuclear genome. The percentage nuclear substitution in females is calculated as $\left(0.5^{n}\right)$, where $n$ is the number of backcross generations. Thus, after seven generations, the amount of the original maternal genome remaining will theoretically be only $7.8 \times 10^{-3}$ percent of the genotype. Although the original genotype is largely replaced by the UjuT genome in these introgressed lines, the maternally inherited Wolbachia infection should remain consistent during the introgressions. The resulting strains were designated $I K 7$ and $I H 7$ for the single- and superinfected introgressed lines, respectively (Table 1).

Following introgressions, crossing experiments designed to characterize Wolbachia effects were conducted using the UjuT, IKT, and IH7 strains. Experimental crosses consisted of 10 2-day-old virgin females and males (20 mosquitoes total) placed in cages. For all crosses, a constant supply of $10 \%$ sucrose was provided to adults. Females were provided a mouse weekly for blood feeding. An oviposition container was constantly available to females and changed weekly for egg collection. Eggs were dried over a 2-day period and then allowed to mature for 5 days at $28 \pm 2{ }^{\circ} \mathrm{C}(80 \pm 5 \%$ $\mathrm{RH})$. Following drying and maturation, eggs were hatched by submerging in a deoxygenated water/liver powder solution. Larvae used for maintenance and crossing experiments were reared at low density in an excess of a yeast/yeast food suspension. Numbers of surviving males and females were recorded weekly. Egg papers were collected and counted until females in the cage were dead. Four replicate cages were monitored simultaneously for each of the nine crossing types.

\section{PCR assay}

Infection type in mosquito strains was initially confirmed using diagnostic primers that amplify a region of the gene encoding the Wolbachia outer surface protein: $w A l b A$ (primers $328 \mathrm{~F}$ and 691R) and $w A l b B$ (183F and 691R primers; Zhou et al, 1998). For samples failing 
Table 1 Aedes albopictus strains used in introgressions and experiments

\begin{tabular}{|c|c|c|c|c|}
\hline Strain designation & Genotype & Wolbachia type & Notes & Reference(s) \\
\hline UjuT & $\mathrm{U}$ & Uninfected & Artificially generated; aposymbiotic & Otsuka and Takaoka (1997) \\
\hline Koh & K & $w \mathrm{AlbA}$ & Naturally occurring; field collected & $\begin{array}{l}\text { Kambhampati et al (1993); Sinkins et al } \\
(1995 a, b)\end{array}$ \\
\hline Hou & $\mathrm{H}$ & $w \mathrm{AlbA}+w \mathrm{AlbB}$ & Naturally occurring; field collected & $\begin{array}{l}\text { Kambhampati et al (1993); Sinkins et al } \\
(1995 a, b)\end{array}$ \\
\hline HT1 & $\mathrm{H}$ & Uninfected & Artificially generated; aposymbiotic & $\begin{array}{l}\text { Dobson et al (2002); Dobson and } \\
\text { Rattanadechakul (2001) }\end{array}$ \\
\hline$I K 7$ & $\mathrm{U}$ & $w \mathrm{AlbA}$ & Artificially generated; introgressed & This paper \\
\hline IH7 & $\mathrm{U}$ & $w \mathrm{AlbA}+w \mathrm{AlbB}$ & Artificially generated; introgressed & This paper \\
\hline
\end{tabular}

to amplify using Wolbachia-specific primers (eg, UjuT strain), 12S primers were used to amplify mitochondria DNA as a positive control for template DNA quality (O'Neill et al, 1992). For PCR amplifications, ovaries or testes from individual mosquitoes were isolated and homogenized in $100 \mu \mathrm{l}$ STE $(0.1 \mathrm{M} \mathrm{NaCl}, 10 \mathrm{mM}$ Tris$\mathrm{HCl}$, and $1 \mathrm{mM}$ EDTA ( $\mathrm{pH}$ 8.0)). Proteinase $\mathrm{K}$ was added to a final concentration of $0.4 \mathrm{mg} / \mathrm{ml}$, and this mixture was incubated at $56^{\circ} \mathrm{C}$ for $1 \mathrm{~h}$. Following heat inactivation at $95^{\circ} \mathrm{C}$ for $15 \mathrm{~min}, 1 \mu \mathrm{l}$ of these samples was amplified in $50 \mathrm{mM} \mathrm{KCl}, 20 \mathrm{mM}$ Tris- $\mathrm{HCl}$ (pH 8.4), $1.5 \mathrm{mM} \mathrm{MgCl}_{2}, 0.25 \mathrm{mM}$ dNTPs, $0.5 \mathrm{mM}$ primers, and $1 \mathrm{U}$ Taq DNA polymerase in a total volume of $20 \mu \mathrm{l}$. Samples were denatured for $3 \mathrm{~min}$ at $94^{\circ} \mathrm{C}$, cycled 35 times at 94 , 55 , and $72{ }^{\circ} \mathrm{C}$ ( $1 \mathrm{~min}$ each), followed by a $10 \mathrm{~min}$ extension at $72^{\circ} \mathrm{C}$ using a PTC-200 Thermal Cycler (MJ Research). A volume of $10 \mu \mathrm{l}$ of each amplification was separated on $1 \%$ agarose gels, stained with ethidium bromide, and visualized under ultraviolet illumination.

\section{Results}

\section{Molecular confirmation of Wolbachia type in introgressed strains}

PCR amplification with primers diagnostic for the wAlbA and wAlbB infections (Zhou et al, 1998; Dobson et al, 2001) confirmed the Wolbachia infection type following introgression crosses. Both the $w \mathrm{AlbA}$ and wAlbB amplification products were observed in $\mathrm{IH7}$ individuals, indicating superinfection. Only the wAlbA amplification product was observed in $I K 7$ individuals. No amplification product was observed in UjuT individuals. This pattern of amplification products is similar to that observed in the original A. albopictus strains (Dobson et al, 2001) and demonstrates that the Wolbachia infection type remained constant throughout introgression crosses.

\section{Cytoplasmic incompatibility}

As shown in Table 2, cytoplasmic incompatibility and reduced egg hatch were observed in crosses only when the male harbored a Wolbachia infection type that was not present in the female mate. Superinfected IH7 females were compatible with all male infection types. Singleinfected $I K 7$ females were incompatible only with superinfected males. Uninfected UjuT females were compatible with uninfected males only. Thus, crosses of the introgressed strains demonstrated an additive pattern of unidirectional cytoplasmic incompatibility. Differences in egg hatch rates (ie, CI levels) were significant by ANOVA and Bonferroni mean separation on $\arcsin (\operatorname{sqrrt}(x))$ transformed data. As illustrated in Figure 2, the egg hatch rate remained relatively constant over time.
Table 2 Percent egg hatch resulting from crosses of introgressed strains (mean \pm standard error)

\begin{tabular}{lrcc}
\hline Female type & \multicolumn{3}{c}{ Male type } \\
\cline { 2 - 4 } & \multicolumn{1}{c}{ IH7 } & IK7 & UjuT \\
\hline IH7 & $90.2 \pm 1.0^{\mathrm{A}}$ & $87.7 \pm 2.0^{\mathrm{A}}$ & $89.7 \pm 1.1^{\mathrm{A}}$ \\
IK7 & $0.0 \pm 0.0^{\mathrm{C}}$ & $86.1 \pm 1.2^{\mathrm{A}}$ & $88.9 \pm 1.9^{\mathrm{A}}$ \\
UjuT & $0.0 \pm 0.0^{\mathrm{C}}$ & $0.2 \pm 0.04^{\mathrm{D}}$ & $73.6 \pm 3.9^{\mathrm{B}}$ \\
\hline
\end{tabular}

Superscripted letters indicate significant differences $(P<0.0001$, $\mathrm{df}=8, \mathrm{~F}=133.0$ ).

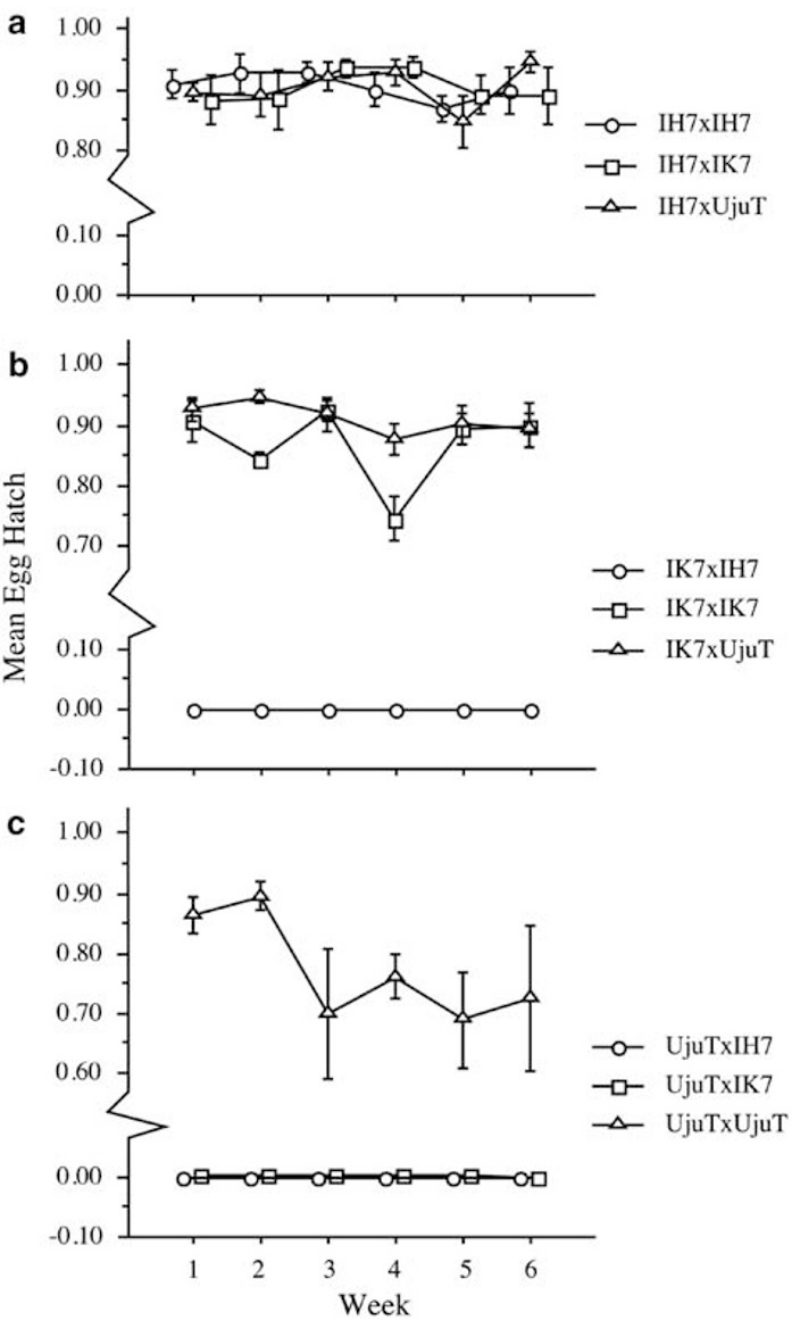

Figure 2 Mean proportion egg hatch rate ( \pm standard error) over time. Crosses are shown as female $\times$ male. Week number indicates time post eclosion. In (a), the symbols are offset for clarity. 
Comparisons within the six compatible cross types demonstrated significantly lower egg hatch resulting from the $U j u T \times U j u T$ cross relative to the remaining five compatible crossing types $(P<0.0001$; Table 2$)$. Egg hatch in compatible crosses of infected females did not differ significantly. In a comparison of the three incompatible cross types, egg hatch was higher in crosses of singleinfected males relative to incompatible crosses of superinfected males $(P<0.0001 ;$ Table 2$)$. No difference in egg hatch was observed between incompatible crosses of superinfected males.

\section{Host longevity}

Differences in host longevity were observed in comparisons of the different Wolbachia infection types. Comparisons were made using two methods. Survivor curves for individual hosts were compared using the Kaplan-Meier method and log-rank test. In a second approach, repeated-measures analysis was used to compare the proportion of surviving individuals in each cage over time. In the latter test, cages were the experimental units and an arcsine transformation of the square root of the proportion was used to ensure near normality of distribution and to stabilize the variance (Gill, 1978). Both analyses yielded the same conclusions. Here, we present the results of the survivor curve analysis.

Comparisons of females with similar infection types demonstrated that the type of male mate did not affect female longevity (IH7 females, $\chi^{2}=4.259, \mathrm{df}=2$, $P>0.119$; IK7 females, $\chi^{2}=3.141, \mathrm{df}=2, P>0.208$; UjuT females, $\left.\chi^{2}=0.215, \mathrm{df}=2, P>0.898\right)$. However, significant differences in female longevity were observed in comparisons of females that were infected with different Wolbachia types. As shown in Figure 3a, superinfected females were longer lived relative to uninfected females $\left(\chi^{2}=24.634, \mathrm{df}=1, P<0.0001\right)$. Single-infected females were also significantly longer lived than uninfected females $\left(\chi^{2}=19.294, \mathrm{df}=1, P<0.0001\right)$. No difference was observed in comparisons of single- and superinfected female longevity $\left(\chi^{2}=0.437, \mathrm{df}=1, P>0.5\right)$. Wolbachia infection type in males was not observed to affect male longevity (Figure $3 \mathrm{~b} ; \mathrm{IH7}, \chi^{2}=4.714, \mathrm{df}=2$, $P>0.095 ; \quad I K 7, \quad \chi^{2}=3.737, \quad \mathrm{df}=2, \quad P>0.154 ;$ UjuT, $\left.\chi^{2}=1.609, \mathrm{df}=2, \stackrel{P}{P}>0.447\right)$.

\section{Wolbachia effect on host fecundity}

Repeated-measures ANOVA was used to compare fecundity. Female oviposition rates were estimated as the number of eggs from a cage divided by the number of surviving females within the cage. Cages and not individual mosquitoes were the experimental units. Comparisons of females with similar infection types demonstrated that the type of male mate did not affect female fecundity ( $\mathrm{IH} 7$ females, $\mathrm{F}=0.178, \mathrm{df}=2, P>0.84$; IK7 females, $\mathrm{F}=0.328, \mathrm{df}=2, P>0.72$; UjuT females, $\mathrm{F}=0.551, \mathrm{df}=2, P>0.59)$. In examining the effect of female Wolbachia infection type on fecundity (Figure 3c), uninfected females produced fewer eggs than singleinfected females $(\mathrm{F}=2.989, \mathrm{df}=2, P<0.0538)$ and superinfected females $(P<0.0321)$. No difference was observed in fecundity comparisons of single- and superinfected females $(P>0.8086)$. Similar results were observed in lifetime fecundity, comparing the different Wolbachia infection types.
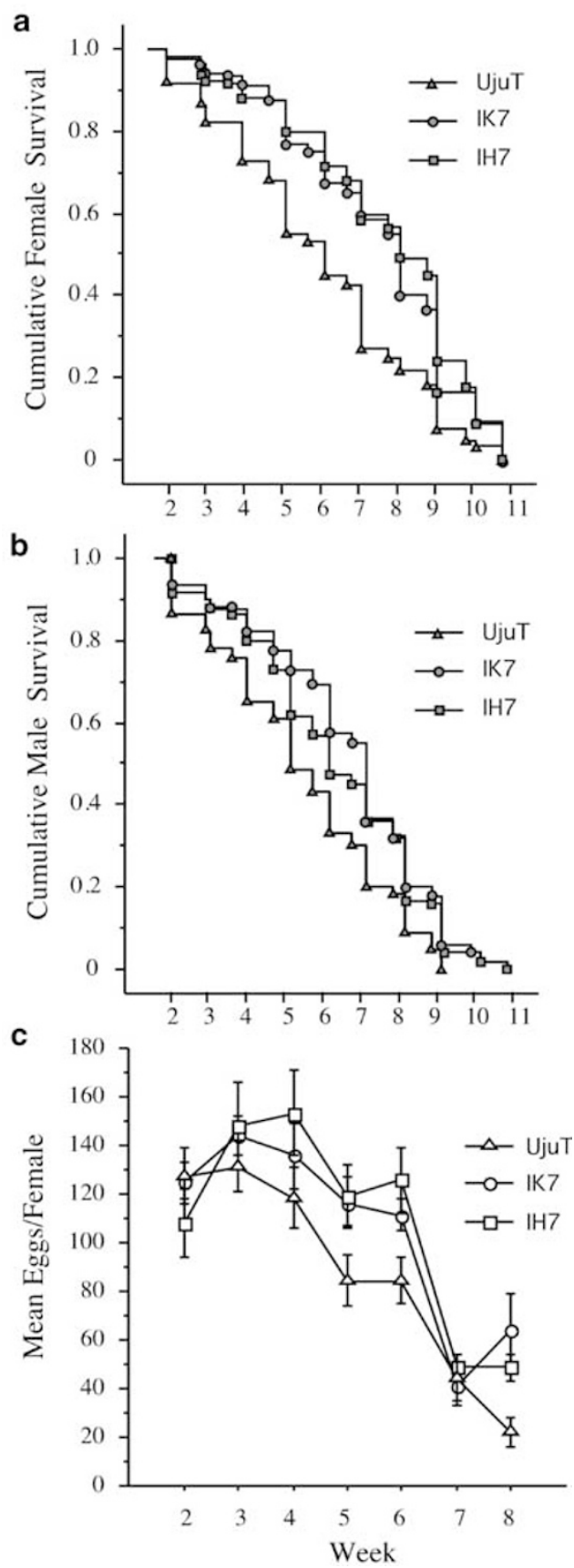

Figure 3 Comparison of (a) female longevity, (b) male longevity, and (c) fecundity by Wolbachia infection type. Week number indicates time post eclosion.

The compatibility type of the cross was not observed to affect fecundity. No difference was observed in comparisons of cytoplasmically incompatible versus compatible crosses of UjuT females $(\mathrm{F}=0.005, \mathrm{df}=1, \quad P>0.94)$. Similarly, no difference was observed in comparisons of cytoplasmically incompatible versus compatible crosses of $I K 7$ females $(\mathrm{F}=0.078, \mathrm{df}=1, P>0.78)$.

Wolbachia infections within differing host genotypes To better define the effect of host genotype on egg hatch, longevity, and fecundity, introgression cross results ('U' genetic background) were compared with results obtained in ' $\mathrm{H}^{\prime}$ genotype crosses (Table 1). As Wolbachia 
infection and compatibility type can affect egg hatch, longevity, and fecundity, comparisons were made between individuals with identical infection types.

To examine host genotype effect on compatible cross egg hatch rates, two cross pairs were compared. In both cross pairs, higher egg hatch was observed in ' $U$ ' genotype mosquitoes. Comparison of a superinfected cross pair $(\mathrm{Hou} \times \mathrm{Hou}$ versus $\mathrm{IH} 7 \times \mathrm{IH} 7)$ demonstrated higher egg hatch $(\mathrm{F}=16.467, \mathrm{df}=1, P<0.001$; Figure 4$)$ in the ' $U$ ' genotype relative to the ' $H$ ' genotype. Higher egg hatch was again observed in the ' $\mathrm{U}$ ' genotype $(\mathrm{F}=7.243$, $\mathrm{df}=1, \quad P<0.0092$; Figure 4) in comparisons of the uninfected $(U j u T \times U j u T ; H T 1 \times H T 1)$ cross pair.

In an examination of genotype effect on fecundity, ' $\mathrm{H}$ ' genotype females were observed to be more fecund than ' $U$ ' genotype females in a comparison of both uninfected $(U j u T \times U j u T ; H T 1 \times H T 1$ cross pair; $\mathrm{F}=48.774, \mathrm{df}=1$, $P<0.0004$; Figure 5a) and superinfected crosses $(\mathrm{Hou} \times \mathrm{Hou}$ vs IH7 $\times \mathrm{IH7} ; \mathrm{F}=4.740, \mathrm{df}=1, \quad \mathrm{P}<0.0724$; Figure 5b).

In uninfected crosses, ' $U$ ' genotype individuals were longer lived relative to ' $\mathrm{H}$ ' genotype individuals. A comparison of female longevity between superinfected females that were of the $\mathrm{H}$ or $\mathrm{U}$ genotype (ie, the Hou and IH7 strains, respectively) demonstrated that $U$ genotype females lived longer $(\mathrm{F}=9.614, \mathrm{df}=1, \quad P<0.0019$; Figure 6a). Similarly, U genotype females were again observed to be longer lived in a comparison of uninfected females $(\mathrm{F}=3.859, \mathrm{df}=1, \quad P<0.0495$; Figure 6b). A comparison of male longevity between males that were of the $\mathrm{H}$ or $\mathrm{U}$ genotype demonstrated that males with the $U$ genotype lived longer regardless of whether superinfected $(\mathrm{F}=3.821, \mathrm{df}=1, \quad P<0.0506$; Figure $6 \mathrm{c})$ or uninfected $(\mathrm{F}=13.420, \mathrm{df}=1, P<0.0002$; Figure 6d).

\section{Discussion}

To improve our understanding of Wolbachia infection dynamics in A. albopictus, we have examined Wolbachia single- and superinfections within a uniform host genetic background generated via introgression crosses. The results demonstrate an increased host fitness associated with Wolbachia infection in A. albopictus. Specifically,

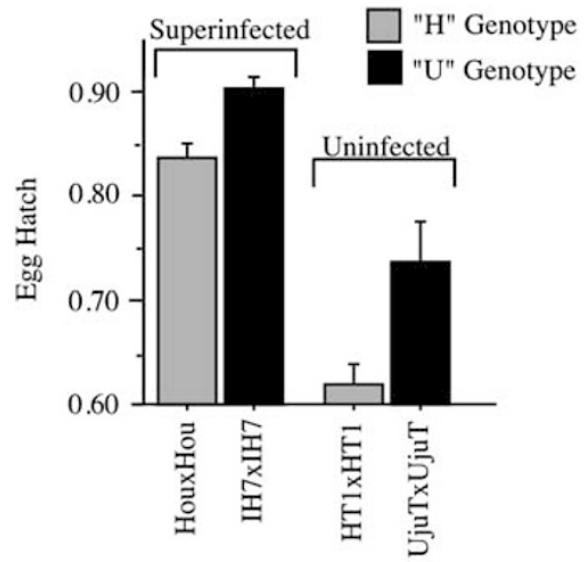

Figure 4 Comparison of host genotype effects on mean proportion egg hatch rate ( \pm standard error), comparing cross pairs of similar infection type. See Table 1 for genotype designation.
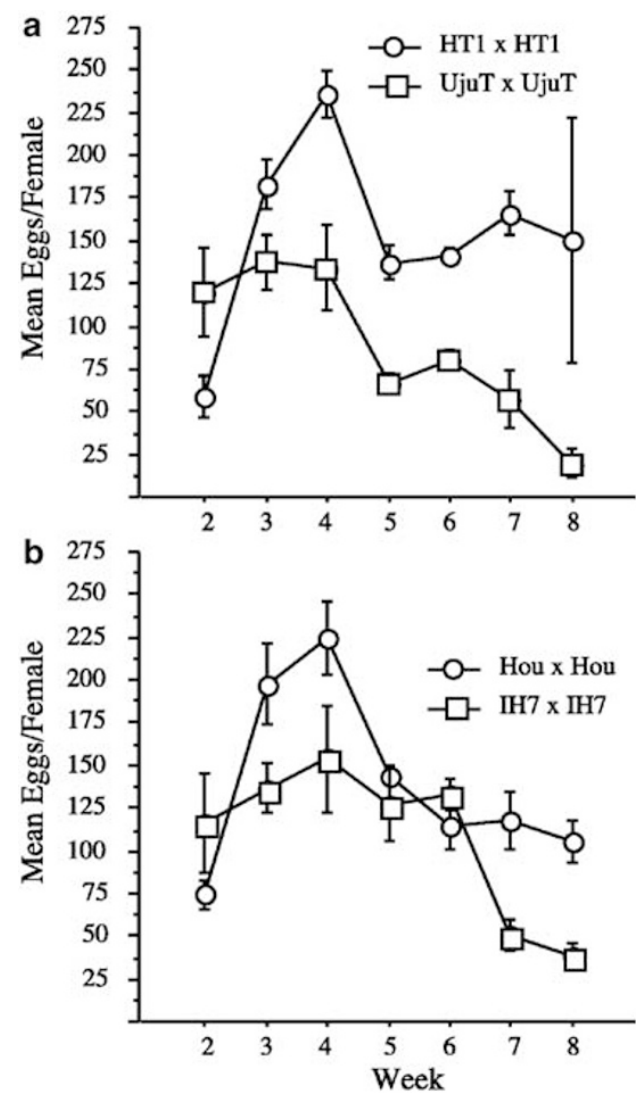

Figure 5 Fecundity comparison of cross pairs in which the host strains differ in genotype but are similarly (a) uninfected or (b) superinfected. Bars indicate standard error. Week number indicates time post eclosion.

single- and superinfected females are longer lived, have higher egg hatch in compatible crosses, and are more fecund relative to uninfected females. In contrast, no fitness differences were observed in comparisons of single- and superinfected mosquitoes. Crosses of the introgressed strains demonstrate an additive pattern of unidirectional cytoplasmic incompatibility consistent with previous reports (Kambhampati et al, 1993; Sinkins et al, 1995b; Otsuka and Takaoka, 1997; Dobson et al, 2001, 2002). Similar to a prior study (Dobson et al, 2001), egg hatch in incompatible crosses of single-infected males was higher than incompatible crosses of superinfected males.

In contrast, male Wolbachia infection type was not observed to affect male longevity. As males are an evolutionary dead end for Wolbachia infections, there is no direct selection on Wolbachia to affect male fitness. Indirectly, Wolbachia will be selected to affect male fitness if the male effect corresponds with a benefit to infections in females (reproductive parasitism; Bandi et al, 2001). Comparisons of introgression crosses also demonstrate that male infection type does not significantly affect egg hatch in compatible crosses, female mate longevity or fecundity. It is important to note that the comparisons described here are limited and do not examine for fitness effects induced by Wolbachia infections in immature hosts and other life stages (e.g., male mating competitiveness).

To examine for host genotype effects on Wolbachia infections, we compared the crossing results of similar 

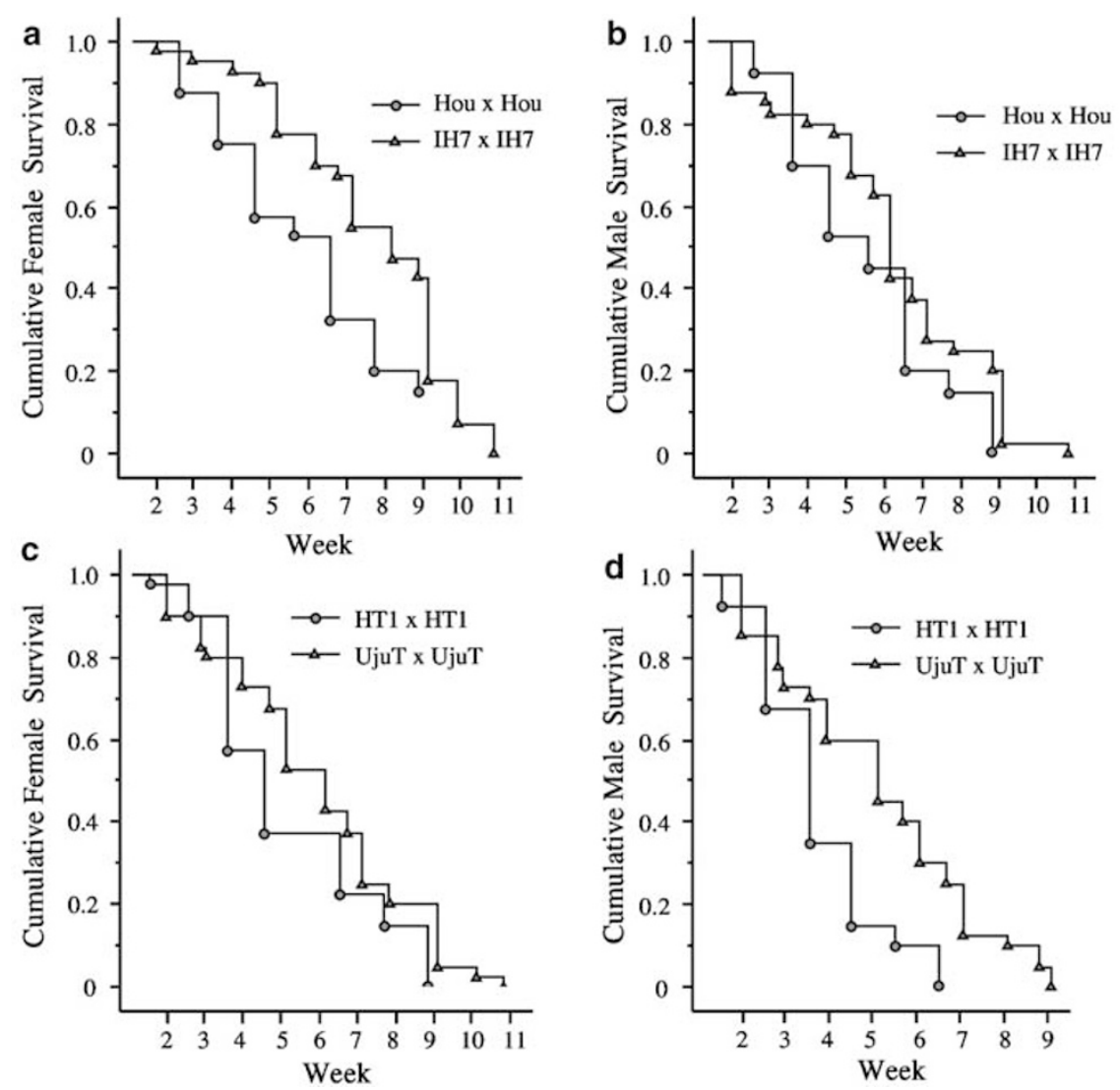

Figure 6 Comparison of host genotype effect on (a and $\mathbf{c}$ ) female and (b and $\mathbf{d}$ ) male longevity using cross pairs in which the host strains differ in genotype but are similarly ( $\mathbf{a}$ and $\mathbf{c}$ ) superinfected or (b and $\mathbf{d}$ ) uninfected. Week number indicates time post eclosion.

Wolbachia infection types within differing genotypes. In general, Wolbachia effects on cytoplasmic incompatibility and host fitness are consistent regardless of host genotype, but differences have been observed in the egg hatch, longevity, and fecundity (Dobson et al, 2001, 2002). This supports the hypothesis that variable host genotype and Wolbachia infection type can complicate the interpretation of crossing results and demonstrate the importance of uniform host genotype in comparisons of Wolbachia infection effects. Given that the UjuT and HT1 strains were cleared of infection in 1997 and 2001, respectively (Otsuka and Takaoka, 1997; Dobson and Rattanadechakul, 2001), the differences between the aposymbiotic UjuT and HT1 strains may reflect differential adaptation of the host mosquitoes to the absence of Wolbachia. Importantly, although introgressions reduced host genetic variability, introgressions did not reduce mitochondrial variability. Thus, mitochondrial variation may contribute to the observed fecundity differences. However, $A$. albopictus displays low mtDNA variation across a wide geographic range (Kambhampati and Rai, 1991; Birungi and Munstermann, 2002). Furthermore, a similar fecundity difference was observed in prior comparisons of superinfected and aposymbiotic strains of $A$. albopictus that did not differ in their mitochondria type (Dobson et al, 2002).

Based upon the observed pattern of additive unidirectional CI, superinfected A. albopictus females are at a reproductive advantage relative to both uninfected and single-infected females. Single-infected females are at a reproductive advantage relative to uninfected females (Figure 1). The Wolbachia infection in A. albopictus is also associated with an increase in fecundity, female longevity, and egg hatch. Here, we observe no difference in female longevity or fecundity in comparisons of singleand superinfected females. Thus, the reproductive advantage of superinfected females relative to singleinfected females is due to cytoplasmic incompatibility only. Therefore, different infection dynamics would be predicted for invasions of Wolbachia single- and superinfections into A. albopictus populations.

As previously demonstrated (reviewed in Hoffmann and Turelli, 1997), the reproductive advantage afforded to infected females by cytoplasmic incompatibility can permit the Wolbachia infection to spread, replacing the cytotype of the host population ('population replacement'). Theoretical predictions are that population replacement will not always result following the introduction of Wolbachia infection into a naive host population (Figure 7a). Due to fecundity costs associated with Wolbachia and the failure of females to transmit the infection to offspring, a minimum threshold infection frequency is required for Wolbachia to spread. Wolbachia occurring at infection frequencies below this minimum threshold are expected to be lost from the host population.

As previously described (Dobson et al, 2002), the combination of $\mathrm{CI}$ and host fitness increase can reduce the threshold infection frequency required for Wolbachia invasion and accelerate the rate of Wolbachia invasion 

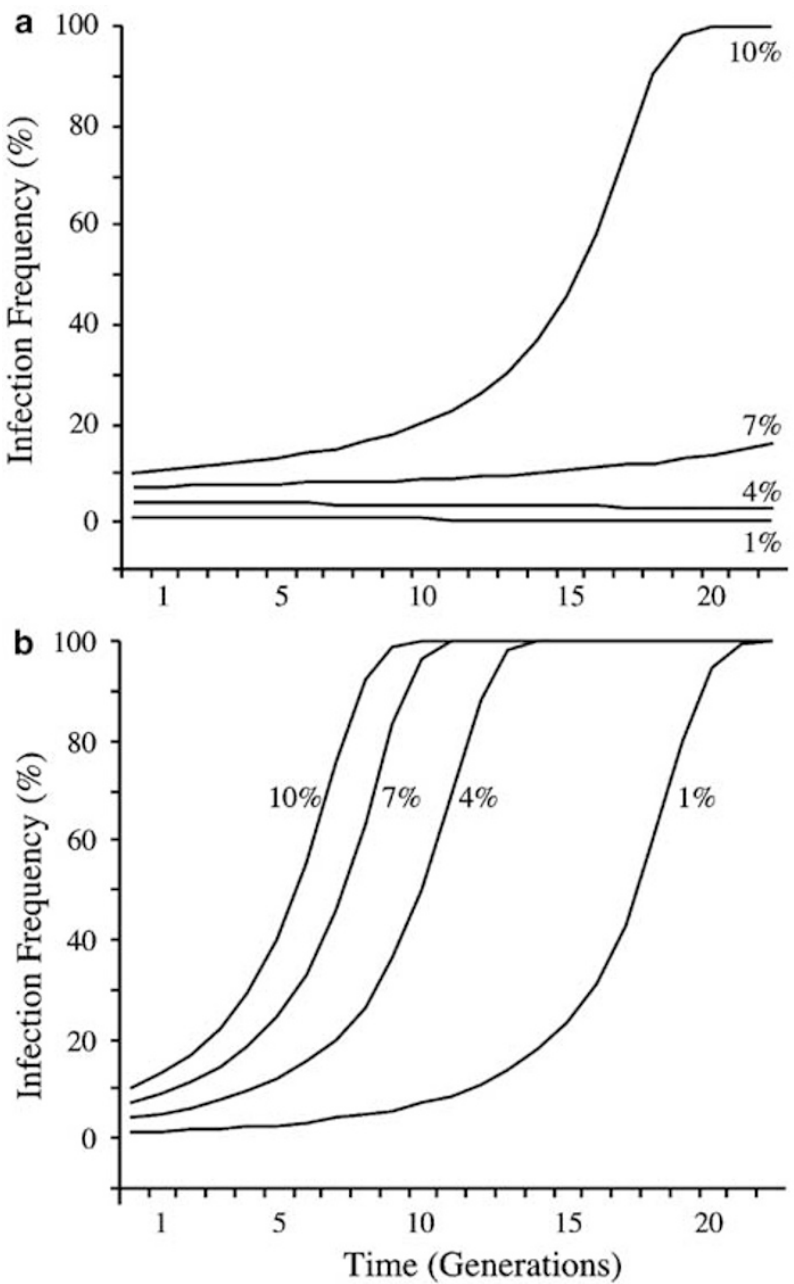

Figure 7 Model predictions of population replacement by (a) Wolbachia infections that cause CI and do not affect host fecundity and (b) Wolbachia infections that both cause CI and increase host fecundity. In both (a) and (b), plotted lines illustrate predicted changes in Wolbachia infection frequency over time following a 1, 4, 7 , and 10\% initial infection frequency. As illustrated in (a), the 1 and $4 \%$ initial infection frequencies are below the infection threshold required for population replacement, resulting in an eventual loss of the Wolbachia infection. In contrast, identical introductions illustrated in (b) result in population replacement. Simulations employ a previously described model (Dobson et al, 2002) and assume a $1 \%$ egg hatch resulting from CI crosses and 95\% maternal transmission of Wolbachia infection. (a) assumes no fecundity difference between the infected and uninfected hosts. (b) assumes $20 \%$ lower fecundity in uninfected hosts.

(ie, cytoplasmic drive rate). Therefore, similar dynamics would be expected for the invasion of an uninfected population by either the single- or superinfections. In both population replacement events, the invasion would be driven by both $\mathrm{CI}$ and a fitness increase associated with Wolbachia infection (Figure $7 \mathrm{~b}$ ). In contrast, a higher threshold infection frequency and slower replacement rate is expected for the invasion of a single-infected population by the Wolbachia superinfection, since it is driven only by CI (Figure 7a).

The threshold infection frequency required for Wolbachia invasion is relevant to the evolution of Wolbachia symbioses and the reported geographic distribution of Wolbachia infections. Specifically, the threshold frequency is relevant to the mechanism by which Wolbachia is introduced into a naive host population. The introduction mechanism must occur at a rate that is sufficient to establish infection in a number of host individuals that surpasses the required threshold Wolbachia infection frequency and permits infection invasion. One mechanism that has been proposed for the introduction of Wolbachia infection into a naive host species is interspecific horizontal transmission. However, based on phylogenetic analyses of Wolbachia infections in insects, naturally occurring interspecific Wolbachia transmission events are predicted to be rare (O'Neill et al, 1992; Rousset et al, 1992; Werren et al, 1995b; Zhou et al, 1998; Vavre et al, 1999). Thus, a reduction of the required threshold by a Wolbachia-associated fitness increase could facilitate the initial invasion of Wolbachia into a naive A. albopictus population following rare interspecific transmission events. Alternatively, the fitness increase associated with Wolbachia infection in $A$. albopictus reported here may have evolved subsequent to the initial symbiotic association and Wolbachia invasion.

The higher threshold required for superinfection invasion of a single-infected population may be related to the geographic distribution of Wolbachia infections in A. albopictus. Superinfection has been described in naturally occurring A. albopictus populations from Japan, India, America, Malaysia, and Thailand (Kambhampati et al, 1993; Otsuka and Takaoka, 1997; Kittayapong et al, 2000). In contrast, naturally occurring wAlbA single infections have only been described in island populations (Koh Samui and Mauritius; Kambhampati et al, 1993; Sinkins et al, 1995b; Kittayapong et al, 2000; Dobson et al, 2001) that are geographically isolated from superinfected populations. Thus, immigration rates of superinfected mosquitoes from the mainland to Koh Samui and Mauritus may have been insufficient to surpass the required threshold. However, mosquito immigration rates are expected to increase with higher human trafficking between the mainland and islands. It should be noted that additional factors including host population structure and Wolbachia maternal inheritance rates are also important in determining the required infection threshold.

Future experiments should include population cage experiments to empirically examine model-predicted population replacement events and examining for additional Wolbachia effects on A. albopictus, including effects in other life stages and effects on mating competition. Experiments examining for potential routes of horizontal transmission of Wolbachia infection into A. albopictus will also help to elucidate the evolutionary trajectory that has resulted in the observed patterns and distribution of Wolbachia/A. albopictus symbiosis.

\section{Acknowledgements}

We thank Charles Fox, Sylvain Charlat, and Claudia Rauter for their comments and suggestions on early versions of the manuscript. We also thank Helena Truszczynska for her aid with statistical analyses. This research was supported in part by the United States Department of Agriculture NRICGP grant \#9902683 and grant AI051533 from the National Institutes of Health. This is publication 02-08-143 of the University of Kentucky Agricultural Experiment Station. 


\section{References}

Bandi C, Dunn AM, Hurst GDD, Rigaud T (2001). Inherited microorganisms, sex-specific virulence and reproductive parasitism. Trends Parasitol 17: 88-94.

Birungi J, Munstermann LE (2002). Genetic structure of Aedes albopictus (Diptera: Culicidae) populations based on mitochondrial ND5 sequences: evidence for an independent invasion into Brazil and United States. Ann Entomol Soc Am 95: 125-132.

Dobson SL, Marsland EJ, Rattanadechakul W (2001). Wolbachiainduced cytoplasmic incompatibility in single- and superinfected Aedes albopictus (Diptera: Culicidae). J Med Entomol 38: 382-387.

Dobson SL, Marsland EJ, Rattanadechakul W (2002). Mutualistic Wolbachia infection in Aedes albopictus: accelerating cytoplasmic drive. Genetics 160: 1087-1094.

Dobson SL, Rattanadechakul W (2001). A novel technique for removing Wolbachia infections from Aedes albopictus (Diptera: Culicidae). J Med Entomol 38: 844-849.

Gerberg EJ, Barnard DR, Ward RA (1994). Manual for mosquito rearing and experimental techniques. American Mosquito Control Association, bulletin \#5 (revised).

Gill JL (1978). Design and Analysis of Experiments in Animal and Medical Sciences. Iowa State University Press: Ames.

Hoerauf A, Nissen-Pahle K, Schmetz C, Henkle-Duhrsen K, Blaxter ML, Buttner DW, Gallin MY, Al-Qaoud KM, Lucius R, Fleischer B (1999). Tetracycline therapy targets intracellular bacteria in the filarial nematode Litomosoides sigmodontis and results in filarial infertility. J Clin Invest 103: 11-18.

Hoffmann AA, Turelli M (1997). Cytoplasmic incompatibility in insects. In: O'Neill SL, Hoffmann AA, Werren JH (eds) Influential Passengers: Inherited Microorganisms and Arthropod Reproduction. Oxford University Press: Oxford, pp 42-80.

Hurst GDD, Jiggins FM, von der Schulenburg JHG, Bertrand D, West SA, Goriacheva II, Zakharov IA, Werren JH, Stouthamer $\mathrm{R}$, Majerus MEN (1999). Male-killing Wolbachia in two species of insect. Proc R Soc Lond (Biol) 266: 735-740.

Jeyaprakash A, Hoy MA (2000). Long PCR improves Wolbachia DNA amplification: wsp sequences found in $76 \%$ of sixtythree arthropod species. Insect Mol Biol 9: 393-405.

Jiggins FM, Bentley JK, Majerus MEN, Hurst GDD (2001). How many species are infected with Wolbachia? Cryptic sex ratio distorters revealed to be common by intensive sampling. Proc $R$ Soc Lond (Biol) 268: 1123-1126.

Kambhampati S, Rai KS (1991). Mitochondrial DNA variation within and among populations of the mosquito Aedes albopictus. Genome 34: 288-292.

Kambhampati S, Rai KS, Burgun SJ (1993). Unidirectional cytoplasmic incompatibility in the mosquito, Aedes albopictus. Evolution 47: 673-677.

Kittayapong P, Baisley KJ, Baimai V, O'Neill SL (2000). Distribution and diversity of Wolbachia infections in south- east Asian mosquitoes (Diptera: Culicidae). J Med Entomol 37: 340-345.

Langworthy NG, Renz A, Mackenstedt U, Henkle Duhrsen K, Bronsvoort MBD, Tanya VN, Donnelly MJ, Trees AJ (2000). Macrofilaricidal activity of tetracycline against the filarial nematode Onchocerca ochengi: elimination of Wolbachia precedes worm death and suggests a dependent relationship. Proc R Soc Lond (Biol) 267: 1063-1069.

O'Neill SL, Giordano R, Colbert AM, Karr TL, Robertson HM (1992). 16S rRNA phylogenetic analysis of the bacterial endosymbionts associated with cytoplasmic incompatibility in insects. Proc Natl Acad Sci USA 89: 2699-2702.

O'Neill SL, Pettigrew M, Sinkins SP, Braig HR, Andreadis TG, Tesh RB (1997). In vitro cultivation of Wolbachia pipientis in an Aedes albopictus cell line. Insect Mol Biol 6: 33-39.

Otsuka Y, Takaoka H (1997). Elimination of Wolbachia pipientis from Aedes albopictus. Med Ent Zool 48: 257-260.

Perrot-Minnot M, Guo LR, Werren JH (1996). Single and double infections with Wolbachia in the parasitic wasp Nasonia vitripennis: effects on compatibility. Genetics 143: 961-972.

Rousset F, Bouchon D, Pintureau B, Juchault P, Solignac M (1992). Wolbachia endosymbionts responsible for various alterations of sexuality in arthropods. Proc $R$ Soc Lond (Biol) 250: 91-98.

Sinkins SP, Braig HR, O'Neill SL (1995a). Wolbachia pipientis: bacterial density and unidirectional cytoplasmic incompatibility between infected populations of Aedes albopictus. Exp Parasit 81: 284-291.

Sinkins SP, Braig HR, O'Neill SL (1995b). Wolbachia superinfections and the expression of cytoplasmic incompatibility. Proc R Soc Lond (Biol) 261: 325-330.

Stouthamer R, Breeuwer JA, Luck RF, Werren JH (1993). Molecular identification of microorganisms associated with parthenogenesis. Nature 361: 66-68.

Tram U, Sullivan W (2002). Role of delayed nuclear envelope breakdown and mitosis in Wolbachia-induced cytoplasmic incompatibility. Science 296: 1124-1126.

Vavre F, Fleury F, Lepetit D, Fouillet P, Bouletreau M (1999). Phylogenetic evidence for horizontal transmission of Wolbachia in host-parasitoid associations. Mol Biol Evol 16: 1711-1723.

Werren JH, Windsor D, Guo LR (1995a). Distribution of Wolbachia among neotropical arthropods. Proc $R$ Soc Lond (Biol) 262: 197-204.

Werren JH, Windsor DM (2000). Wolbachia infection frequencies in insects: evidence of a global equilibrium? Proc $R$ Soc Lond (Biol) 267: 1277-1285.

Werren JH, Zhang W, Guo LR (1995b). Evolution and phylogeny of Wolbachia: reproductive parasites of arthropods. Proc R Soc Lond (Biol) 261: 55-63.

Zhou W, Rousset F, O'Neill SL (1998). Phylogeny and PCR based classification of Wolbachia strains using wsp gene sequences. Proc R Soc Lond (Biol) 265: 509-515. 